\title{
Effective and persistent antitumor activity of HER2-directed CAR-T cells against gastric cancer cells in vitro and xenotransplanted tumors in vivo
}

\author{
Yanjing Song ${ }^{1,2}$, Chuan Tong ${ }^{3}$, Yao Wang ${ }^{3}$, Yunhe Gao ${ }^{1,2}$, Hanren Dai $^{3}$, Yelei Guo ${ }^{3}$, Xudong Zhao ${ }^{1,2}$, \\ Yi Wang ${ }^{1,2}$, Zizheng Wang ${ }^{2}$, Weidong Han ${ }^{3,4 \bowtie}$, Lin Chen ${ }^{1 凶}$ \\ ${ }^{1}$ Department of General Surgery, Chinese PLA General Hospital, Beijing 100853, China \\ ${ }^{2}$ Medical School of Chinese PLA, Beijing 100853, China \\ ${ }^{3}$ Bio-therapeutic Department, Chinese PLA General Hospital, Beijing 100853, China \\ ${ }^{4}$ Molecular \& Immunology Department, Chinese PLA General Hospital, Beijing 100853, China \\ $\triangle$ Correspondence: hanwdrsw69@yahoo.com (W. Han), chenlinbj@vip.sina.com (L. Chen) \\ Received December 20, 2016 Accepted January 31, 2017
}

\begin{abstract}
Human epidermal growth factor receptor 2 (HER2) proteins are overexpressed in a high proportion of gastric cancer (GC) cases and affect the maintenance of cancer stem cell (CSC) subpopulations, which are used as targets for the clinical treatment of patients with HER2positive GC. Despite improvements in survival, numerous HER2-positive patients fail treatment with trastuzumab, highlighting the need for more effective therapies. In this study, we generated a novel type of genetically modified human $\mathrm{T}$ cells, expressing a chimeric antigen receptor (CAR), and targeting the GC cell antigen HER2, which harbors the CD137 and $C D 3 \zeta$ moieties. Our findings show that the expanded CAR-T cells, expressing an increased central memory phenotype, were activated by the specific recognition of HER2 antigens in an MHC-independent manner, and effectively killed patient-derived HER2-positive GC cells. In HER2-positive xenograft tumors, CAR-T cells exhibited considerably enhanced tumor inhibition ability, long-term survival, and homing to
\end{abstract}

Yanjing Song, Chuan Tong, Yao Wang, Yunhe Gao, Weidong Han and Lin Chen contributed equally to this work.

Electronic supplementary material The online version of this article (doi:10.1007/s13238-017-0384-8) contains supplementary material, which is available to authorized users. targets, compared with those of non-transduced T cells. The sphere-forming ability and in vivo tumorigenicity of patient-derived gastric cancer stem-like cells, expressing HER2 and the CD44 protein, were also inhibited. Our results support the future development and clinical application of this adoptive immunotherapy in patients with HER2-positive advanced GC.

KEYWORDS chimeric antigen receptor, HER2, gastric cancer, cancer stem cell, CD137, immunotherapy

\section{INTRODUCTION}

Gastric cancer (GC) is one of the most common malignant cancers worldwide, especially in China. GC-associated morbidity and mortality, estimated by the National Central Cancer Registry of China (NCCR), ranks second among cancers, and resulted in approximately 679 thousand deaths and 498 thousand new cases in 2015 (Chen et al., 2016). Despite improvements in GC therapy, many patients fail treatment because of tumor recurrence and metastasis. As the predominant treatment for advanced gastric cancer (AGC), chemotherapy is inefficient and toxic because of its nonspecific antitumor activity. Even among patients treated with first-line chemotherapy, the overall response rate (ORR) is less than $50 \%$, and the median overall survival (mOS) is only 6.6 to 18.4 months (Ter Veer et al., 2016). Thus, we focused on adoptive cellular immunotherapy $(\mathrm{ACl})$, which involves the use of genetically engineered T lymphocyte or 
NK cell treatments for hematological malignancies (Porter et al., 2011).

Chimeric antigen receptor (CAR) T-cell immunotherapy is an $\mathrm{ACl}$ in which the CAR-redirected $\mathrm{T}$ cells, expressing engineered receptors specific to a particular antigen, are reintroduced into patients and elicit an effective antitumor immune response. CAR-T cells have been in development for 2 decades, beginning with first-generation chimeric genes composed of single-chain variable fragments (scFvs) of monoclonal antibody (mAb) and FcR $\mathrm{y}$ chains or TCR/ CD3 $\zeta$ chains (Eshhar et al., 1993). Second- and third-generation, with one or more costimulatory molecules, such as CD28, CD137 (4-1BB), ICOS, and OX40, have since been developed. CD19-targeted CAR-T cells display high antitumor activity in children and adults with B-cell malignancies (Park et al., 2016). Additionally, a series of CARs, targeting different antigens, have been constructed and validated in clinical trials. Although this strategy has been effective and presents advantages, such as high specificity and MHC-independent effects, its potential clinical application in solid tumors is hampered by concerns over its safety and efficacy.

Studies on the use of CAR-T cells in the treatment of GC are lacking, mainly because of the high heterogeneity of GC cells. Positive clinical outcomes, obtained using the targeted agent trastuzumab, suggest that the surface antigen HER2 may serve as a target for CAR-T cell therapy. HER2, also known as CD340 or Neu, is a member of the EGFR family, and is encoded by the ERBB2 proto-oncogene. ERBB2 is faintly expressed in the luminal and glandular epithelium under normal conditions and is overexpressed in carcinomas of the breast, ovary, endometrium, lung, pancreas, bladder, and stomach (Thibault et al., 2013). Positive rates of HER2 amplification, and overexpression in GC patients, are distinct and range from $10 \%$ to $27 \%$, and $8.2 \%$ to $53.4 \%$, respectively; this is likely due to differences in methodologies, ethnic groups, pathological types, and tumor locations among the affected patients (Vakiani, 2015). Additionally, ERBB2 amplification contributes to the maintenance of stem cell (GCSC) subpopulations of gastric cancer (Jiang et al., 2012); the expression status of $E R B B 2$ is related to disease progression and poor prognosis (Van Cutsem et al., 2016). In this study, we designed novel, lentivirus-mediated, CAR harboring, anti-HER2 scFv, CD3 $\zeta$ and CD137 signaling domains and evaluated the antitumor activity of CAR-modified T cells against primary GC cells and GCSCs in vitro and in vivo. Our results support future clinical trials testing the use of this CAR-modified T-cell immunotherapy in patients with $A G C$ and multi-drug resistance.

\section{RESULTS}

Identification and characterization of novel HER2targeted CAR-T cells

A second-generation CAR, consisting of an HER2-specific scFv linked to a hinge domain, a transmembrane (TM) domain, and $C D 137 \zeta$ and $C D 3 \zeta$ moieties in sequence (Fig. 1A), was constructed and inserted into a pseudotyped, clinical-grade lentiviral vector system. A green fluorescence protein (GFP) was added to CAR.HER2-CD137ろ to verify transfection efficiency; CAR-CD137ろ-GFP, without an ScFv, was constructed to serve as control.

T cells were originally generated from the peripheral blood mononuclear cells (PBMCs) of three healthy donors. Lentivirus-mediated CAR gene transfection was performed on day 2 of PBMC culture in vitro. The total numbers of CARtransduced, mock-transduced, and non-transduced (NT) cells were expanded on an average of 85,75 , and 90 times, respectively, on day 12 of culture (Fig. 1B). Using fluorescence-activated cell sorting (FACS) analysis on day 12, we determined that the CAR group mainly comprised a large population of $\mathrm{CD}^{+} \mathrm{CD}^{-} 6^{-}$cells $(72.55 \% \pm 7.26 \%)$ and $\mathrm{a}$ small fraction of cells expressing the indicated central memory $\mathrm{T}(\mathrm{Tcm})$ phenotypes $\left(\mathrm{CD} 45 \mathrm{RO}^{+} \mathrm{CD} 62 \mathrm{~L}^{+} \mathrm{CCR} 7^{+}\right.$, $23.35 \% \pm 7.59 \%$ ). The $\mathrm{Tcm}$ population was significantly larger than the corresponding population in the NT group (Figs. 1C and S1). Mean transfection efficiencies of $34.22 \%$ $\pm 4.00 \%$ and $32.95 \% \pm 4.76 \%$ were verified in the final CART-HER2 and mock T-cell populations, respectively (Figs. 1D and S2). Groups of cells, exhibiting optimal growth, were selected from one of the three abovementioned donors, to undergo subsequent functional assays.

\section{CART-HER2 cells specifically kill HER2 ${ }^{+}$GC cell lines and primary GC cells ex vivo}

FACS was used to assess the surface expression of HER2 proteins in a series of human GC cell lines (GCCLs), including the N87, 7901, AGS, HGC27, MGC803, BGC823, and MKN45 cells, and in primary gastric cancer cells (PGCCs) obtained from two patients with GC. HER2 was strongly expressed in all the GCCLs, with percentages ranging from $84.39 \%$ to $98.60 \%$. In contrast, the PGCCs from patient 1 (PGCC 1) exhibited a percentage of $56.80 \%$, and the PGCCs from patient 2 (PGCC 2) exhibited a percentage of $2.48 \%$ (Fig. 2A). Therefore, two different GCCLs (N87 and 7901), and two patient-derived cell lines, were selected for further study. HER2 knockdown (HER2 ${ }^{\mathrm{KD}}$ ) was performed in N87 and 7901 cells via transduction of a lentivirus-mediated short hairpin RNA-HER2 (shRNA-HER2) (Fig. 2B). Transduction of the GFP gene was performed for isolation and tracking. HER2 ${ }^{\mathrm{KD}}$ tumor cells served as control cells.

To verify the specific activity of CART-HER2 cells against $\mathrm{HER}^{+}{ }^{+}$tumor cells, we incubated T cells with tumor cells at an effector-to-target $(E / T)$ ratio of $20: 1$ for $4 \mathrm{~h}$. After the incubation, the levels of cytokines released by CART-HER2 cells, including IL-2, IFN- $\gamma$, IL-4, GM-CSF, TNF- $\alpha$, and Granzyme B (GZMB), were significantly elevated in the supernatants of HER2 ${ }^{\text {high+ }} \mathrm{N} 87$ and 7901 cells compared with those of mock T cells and NT T cells (Fig. 2C). However, 
A
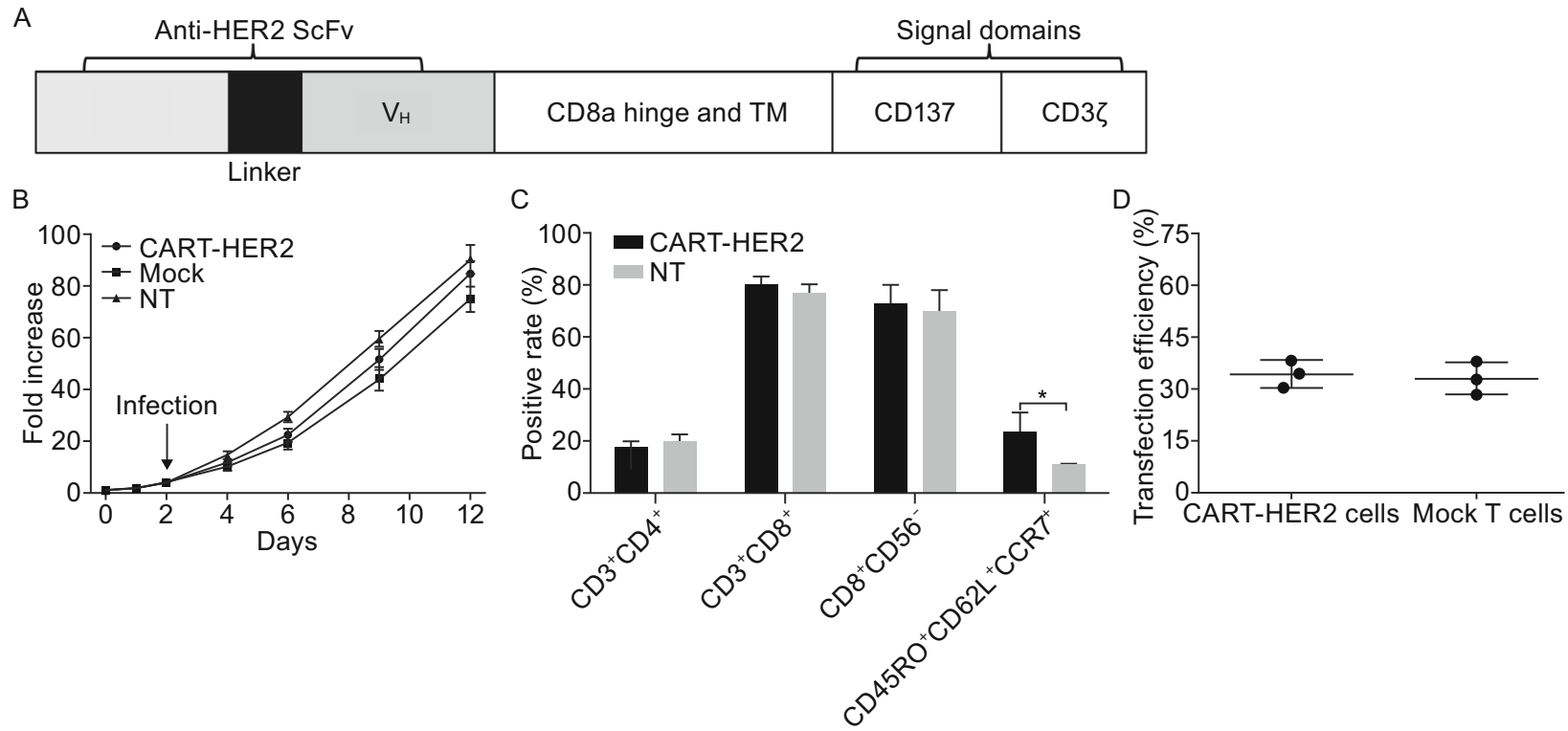

Figure 1. Generation, expansion, and characterization of HER2-directed chimeric antigen receptor $\mathrm{T}$ cells. (A) Schematic representation of anti-HER2 CAR gene sequence (not drawn to scale). (B) Average expansion (-fold) of CART-HER2, mock T, and NT T cells, produced using PBMCs from three healthy donors; the cells were cultured in vitro for 10-14 days. Mean and SDs are shown for three different T-cell lines. (C) Phenotypic features of CART-HER2 and NT T cells, from three healthy donors, were evaluated by FACS analysis on day 12 of culture. Mean positive rates \pm SD from three different T-cell lines are shown. (D) Transfection efficiency of CAR or mock gene into T cells was determined by FACS analysis using the marker GFP on day 12 . The data are represented as means $\pm \mathrm{SD}$. *Represents $P<0.05$.

substantially lower cytokine levels were detected when CART-HER2 cells were co-cultured with HER2 ${ }^{\mathrm{KD}} \mathrm{N} 87$ and 7901 cells than when CART-HER2 cells were co-cultured with HER2 ${ }^{\text {hight }}$ N87 and 7901 cells (Fig. 2C). Similarly, the specific activity of CART-HER2 cells against patient-derived GC cells was verified by incubating the T cells with PGCC 1 . Furthermore, minimal levels of cytokines were detected when effector T cells interacted with HER2- PGCC 2 (Fig. 2D). These results indicate that CART-HER2 cells can specifically recognize $\mathrm{GC}$ cells with high and low expression of HER2, and can then be activated at varying levels by GC cells.

Next, to evaluate the cytotoxicity of CART-HER2 cells against GC cells, we performed time- and dose-dependent lactate dehydrogenase (LDH) release assays. As shown in Fig. $3 \mathrm{~A}$ and $3 \mathrm{~B}$, CART-HER2 cells, evaluated using the 4-h LDH assay, and incubated at a ratio of 20:1 E/T, exhibited higher average killing activity against $\mathrm{HER} 2^{\text {hight }} \mathrm{N} 87$ cells $(65.3 \% \pm 5.0 \%), 7901$ cells $(81.5 \% \pm 2.5 \%)$, and PGCC 1 $(53.6 \% \pm 4.2 \%)$ than did mock and NT T cells having a low rate of lysis. Moreover, the cytotoxicity of CART-HER2 cells was increased as the E/T ratio increased (Fig. 3A). Similarly, the 8-h LDH assay showed that the average killing activity, exhibited by CART-HER2 cells against HER2 ${ }^{+}$GC cells when incubated at a ratio of 20:1 E/T, was higher than that exhibited by mock or NT T cells (Fig. $3 \mathrm{C}$ ), but was not significantly higher than that exhibited by CART-HER2 cells in the 4-h assay (Fig. 3D). In the control group of target cells, the average killing activity levels, exhibited by CART-HER2 cells against HER2 ${ }^{\mathrm{KD}} \mathrm{N} 87$ and 7901 cells during the 4-h $\mathrm{LDH}$ assay when incubated at a ratio of $20: 1 \mathrm{E} / \mathrm{T}$, were $33.6 \% \pm 3.0 \%$ and $45.3 \% \pm 2.2 \%$, respectively; these levels were still significantly higher than those exhibited by mock or NT T cells (Fig. 3B). There was no significant difference among the three types of effector $T$ cells with respect to cytotoxicity against HER2- PGCC 2 (Fig. 3A). Hence, we demonstrated that CART-HER2 cells possess potent cytotoxic activity against $\mathrm{HER}{ }^{+} \mathrm{GC}$ cells.

CART-HER2 cells exhibit effective and persistent antitumor activity against xenografts derived from HER2 ${ }^{+}$GCCLs in mice

The subcutaneous xenotransplanted tumor model of HER2 ${ }^{\text {hight }} 7901$ cells was established in BALB/c nude mice to determine the antitumor ability of CART-HER2 cells in vivo. Treatment with effector cells was performed on day 12, when the mean tumor volume (TV) had reached approximately $100 \mathrm{~mm}^{3}$ after subaxillary inoculation with tumor cells. Tumor growth was measured twice weekly until the maximum tumor diameter was greater than $2 \mathrm{~cm}$ or until $20 \%$ of body weight was lost; the survival time of mice was 

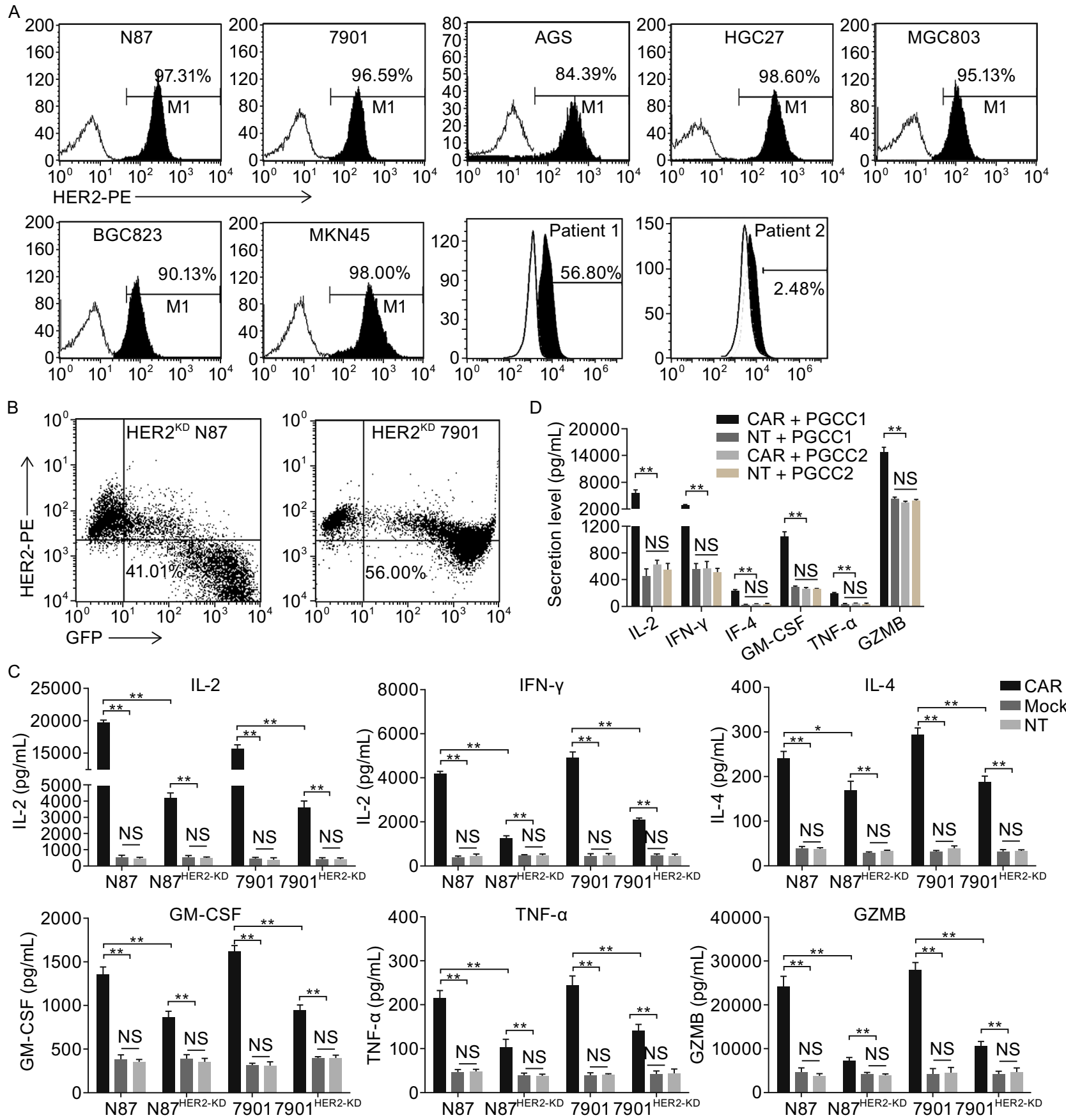

Figure 2. Specific activity of HER2-directed chimeric antigen receptor T cells against HER2 ${ }^{+}$GC cells. (A) FACS was used to test the surface expression of HER2 proteins in a series of human GC cell lines, including N87, 7901, AGS, HGC27, MGC803, BGC823, MKN45, and primary GC cells from two patients with GC. (B) HER2 expression in N87 and 7901 cells was downregulated via transduction of lentivirus-mediated short hairpin RNA-HER2. The knockdown effects of HER2 expression in sorted GFP-positive cells were evaluated by FACS analysis. (C) The levels of cytokines, released by CART-HER2, mock T, and NT T cells, were measured by enzyme-linked immunosorbent assay (ELISA) after 4-h incubation with HER2 ${ }^{\text {hight }}$ and HER2 ${ }^{\mathrm{KD}} \mathrm{GC}$ cells at an effectorto-target (E/T) ratio of 20:1. (D) The levels of cytokines, released by CART-HER2 and NT T cells, were measured by ELISA after 4-h incubation with patient-derived GC cells at an E/T ratio of 20:1. The data are represented as the mean cytokine concentrations \pm SD $(\mathrm{pg} / \mathrm{mL})$ from triplicate cultures. NS represents no statistical significance, *represents $P<0.05$, ${ }^{\star *}$ represents $P<0.01$. 


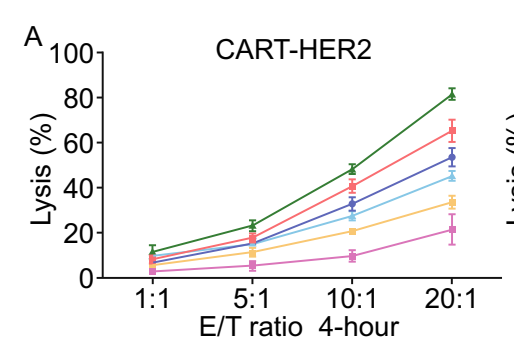

B

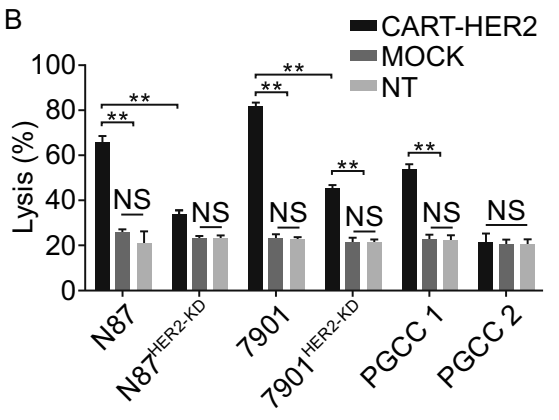

$E: T=20: 1$, 4-hour
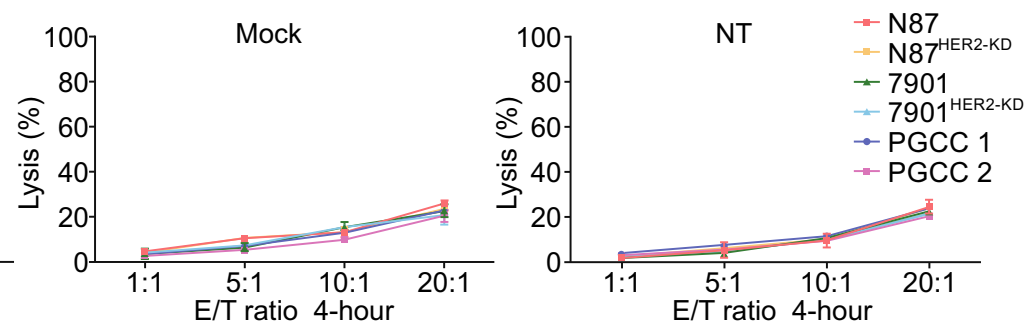

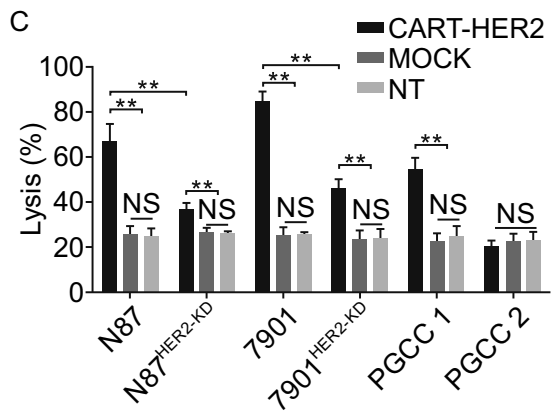

$\mathrm{E}: \mathrm{T}=20: 1$, 8-hour

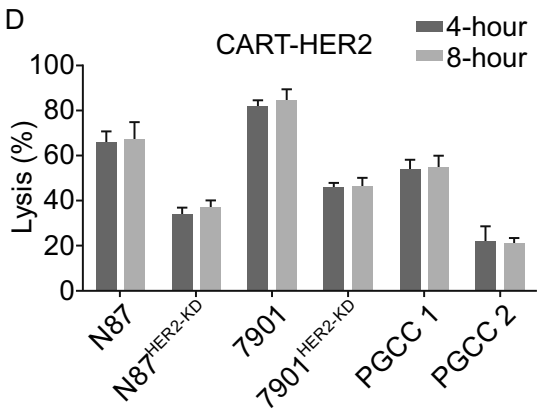

$\mathrm{E}: \mathrm{T}=20: 1$

Figure 3. Specific cytotoxicity exhibited by HER2-directed chimeric antigen receptor T cells against HER2 ${ }^{+}$GC cell line and primary GC cells ex vivo. (A) The cytotoxic activity of CART-HER2, mock T, and NT T cells against HER2 $2^{\text {hight }}$ and HER2 ${ }^{\mathrm{KD}} \mathrm{GC}$ cells was determined using a 4-h lactate dehydrogenase (LDH) release assay in a dose-dependent manner. HER2 ${ }^{-}$primary GC cells were used as controls. (B) A 4-h LDH release assay, at an effector-to-target (E/T) ratio of 20:1, was used to compare cytotoxicity between CART-HER2, mock T, and NT T cells against different GC cells (C) An 8-h LDH release assay, at an E/T ratio of 20:1, was used to compare cytotoxicity between CART-HER2, mock T, and NT T cells against different GC cells. (D) Cytotoxicity comparisons of CARTHER2 cells against different GC cells were performed between the 4-h and 8-h LDH release assays at an E/T ratio of 20:1. All of the results are expressed as the mean of triplicate values \pm SD. NS represents no statistical significance, ${ }^{* *}$ represents $P<0.01$.

over 90 days. As shown in Figs. $4 \mathrm{~A}$ and $\mathrm{S} 3$, in the HER2 ${ }^{\text {high+ }}$ mouse model, the rates of tumor growth were considerably inhibited by treatment with CART-HER2 cells, while tumors in the control group continued to grow rapidly after the injection with NT T cells. The mean TV and mean tumor weight (TW) in the CART-HER2 group were $654 \pm 247 \mathrm{~mm}^{3}$ $(P<0.01)$ and $426 \pm 138 \mathrm{mg}(P<0.01)$, respectively, while those in the NT group reached $1,769 \pm 462 \mathrm{~mm}^{3}$ and $1,050 \pm$ $306 \mathrm{mg}$, respectively, on day 32 . Finally, the significantly improved survival ( $77 \pm 11$ days), exhibited by the remaining HER2 $2^{\text {hight }}$ tumor-bearing mice $(n=3)$ treated with CARTHER2 cells, indicated the specific antitumor activity of CARTHER2 cells.

To determine the persistence of the CART-HER2 cells, we used qPCR at serial time points to measure the copy numbers of $C A R$ in the peripheral blood of mice in the experimental group. The copy numbers of $C A R$ remained at a detectable level for at least 56 days in the blood of the remaining three mice and were positively correlated with the improved survival of HER2 ${ }^{\text {hight }}$ mice (Fig. 4B).

To assess the homing ability of CART-HER2 cells, transgene copy number detection, as well as hematoxylineosin (HE) staining, and immunohistochemical (IHC) labeling, were performed on tumor samples from sacrificed mice. The results showed high levels of DNA copy numbers
(Fig. 4C) and considerable increases in the recruitment of human $\mathrm{CD}^{+} \mathrm{T}$ cells in the experimental group, whereas only a small number of $\mathrm{CD}^{+}$T cells were observed in the NT group (Fig. 4D). These findings strongly suggest that CARTHER2 cells can effectively traffic to target sites.

\section{CART-HER2 cells effectively respond to GCSCs}

To verify whether CART-HER2 cells can inhibit the growth of CSC subpopulations in primary GC, suspended cell spheres, which are aggregations of CSCs, were generated in serumfree media containing growth factors. The mean number of spheres per well, in an ultra-low adherent 6-well plate, was 32 on day 20 after inoculation with HER2 ${ }^{+}$PGCCs from patient 1. The rates of the surface expression of HER2, and the GCSCs-specific marker CD44, in separated spheres were $73.5 \%$ and $98.6 \%$, respectively, as determined by FACS analysis (Fig. 5A). Then, a co-culture of tumor spheres with $2 \times 10^{5}$ CART-HER2 cells or NT T cells per well, was grown in an ultra-low adherent 24-well plate using serumfree media. CART-HER2 cells migrated to the tumor spheres by chemotaxis after $6 \mathrm{~h}$ of co-culture, resulting in the phagocytosis and degradation of the tumor spheres by the CART-HER2 cells. The numbers of spheres in five wells fell by an average of $50 \%$ after $24 \mathrm{~h}$. Most of the spheres in the 

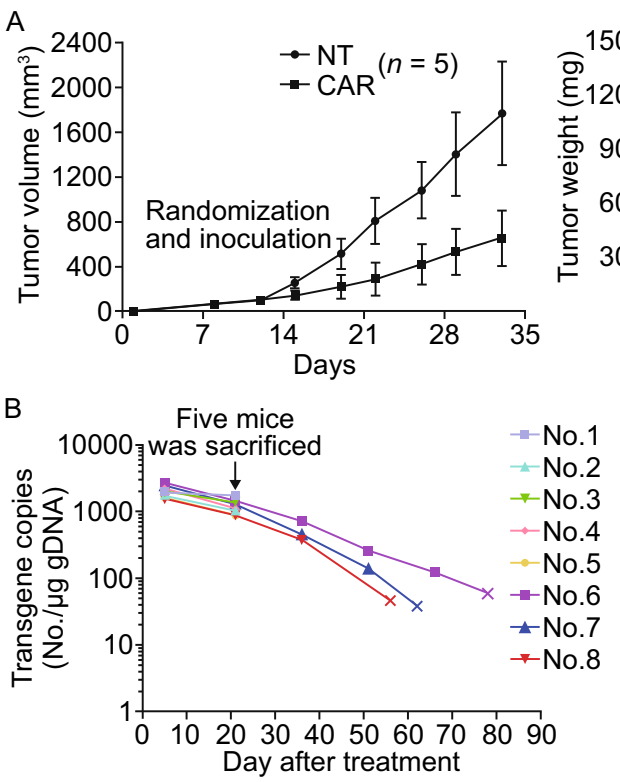

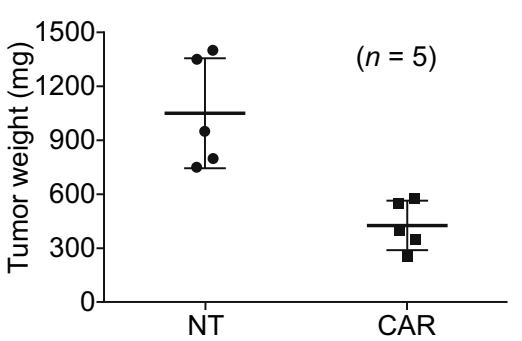

C

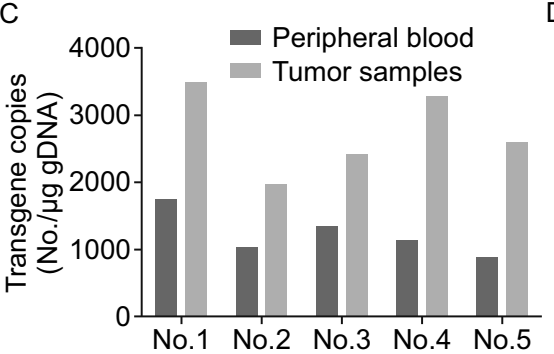

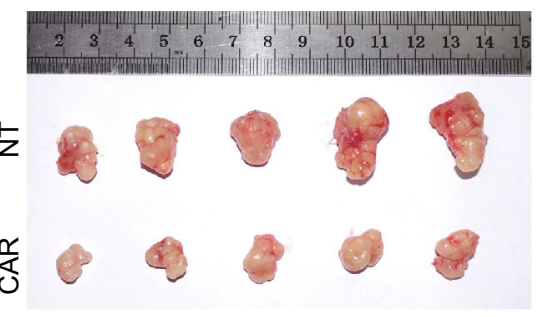

D
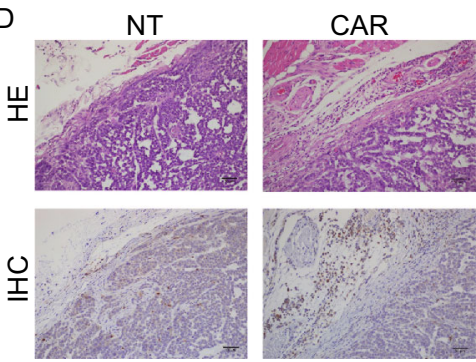

Figure 4. Antitumor efficacy of HER2-directed chimeric antigen receptor T cells against xenografts derived from HER2 ${ }^{+} \mathrm{GC}$ cell lines in mice. (A) Two million HER2 ${ }^{\text {hight }} 7901$ cells were injected subcutaneously into the unilateral axillary region of BALB/c nude mice on day 0 . Five HER2 $2^{\text {hight }}$ tumor-bearing mice per group were randomly assigned to the CART-HER2 and NT groups before treatment. When the mean tumor volume reached approximately $100 \mathrm{~mm}^{3}$ on day 12 , intravenous treatment with T cells was performed. Mice with maximum tumor diameters over $2 \mathrm{~cm}$ were sacrificed on day 33. The tumor volume data are represented as mean $\pm \mathrm{SD}\left(\mathrm{mm}^{3}\right)$. The tumor weight data are represented as mean $\pm \mathrm{SD}(\mathrm{mg})$. Images of tumor samples from sacrificed mice are shown. (B) The persistence of the infused CART-HER2 cells in the peripheral blood. qPCR was performed at serial time points, after infusion with CART-HER2 cells, to determine the expression levels of CAR in the peripheral blood of mice $(n=8)$. (C) Correlations of CAR copy numbers in tumor tissue and blood samples, obtained after the HER2 ${ }^{\text {hight }}{ }^{\text {mice, }}$ treated with CART-HER2 cells, were sacrificed on day 33. (D) Hematoxylin-eosin (HE) and immunohistochemical (IHC) staining for anti-CD3 were performed on tumor samples from sacrificed mice.

CART-HER group were disintegrated into small pieces after $48 \mathrm{~h}$, indicating that the CART-HER2 cells were significantly more effective than the NT T cells at targeting the tumor spheres (Fig. 5B). The tumorigenicity of the CSCs in the $B A L B / c$ nude mice was also remarkably restrained by intravenous treatment with CART-HER2 cells. The average TV and TW in the CART-HER2 group were $450 \pm 106 \mathrm{~mm}^{3}$ and $310 \pm 81 \mathrm{mg}$, respectively, while those in the NT group were $1,377 \pm 196 \mathrm{~mm}^{3}$ and $852 \pm 124 \mathrm{mg}$, respectively, on day 63 (Figs. $5 \mathrm{C}$ and S4).

\section{DISCUSSION}

To the best of our knowledge, this study is the first to confirm that CART-HER2 cells show specific, effective, and persistent tumor-killing activity against HER2-positive primary GC cells and GCSCs in pre-clinical tests. We designed an antiHER2 scFv and CD137-containing CAR, which was transduced into $T$ cells by a clinical-grade lentiviral vector system. Our results show that the cytotoxicity, exhibited by CARTHER2 cells against HER2-positive GC cells and HER2knockdown cells, was significantly higher than that exhibited by NT T cells; this rules out the influence of the vector system on our results and suggests that CAR-T cells contacted the target cells with high affinity and lysed them in an MHCindependent manner. The secretion levels of multiple cytokines were positively correlated with the antitumor effects of CAR-T cells. The cytokines that were upregulated included the pro-inflammatory factors IFN- $\gamma$, IL-2, IL-4, GMCSF, and TNF- $\alpha$, which are crucial for T-cell activation, proliferation, and differentiation. These cytokines are also implicated in the occurrence of severe cytokine release syndrome (CRS) (Teachey et al., 2016). HER2-positive tumor growth in mice was markedly inhibited by intravenous infusion of CAR-T cells. We detected persistently high copy numbers of $C A R$ in the blood and high numbers of infiltrating $\mathrm{CD}^{+}$cells in tumors, all of which indicated the persistence, homing, and antitumor activity of CAR-T cells. The sphereforming ability, and in vivo tumorigenicity, of primary GCSCs were significantly suppressed by treatment with CAR-T cells; thus, we conclude that CART-HER2 cells can mount an effective response to CSCs.

Components of the HER2 signaling pathway have been used extensively as a key immunotherapy target in therapy against various solid tumors. Although several anti-HER2 drugs, such as trastuzumab, pertuzumab, lapatinib, and 
A
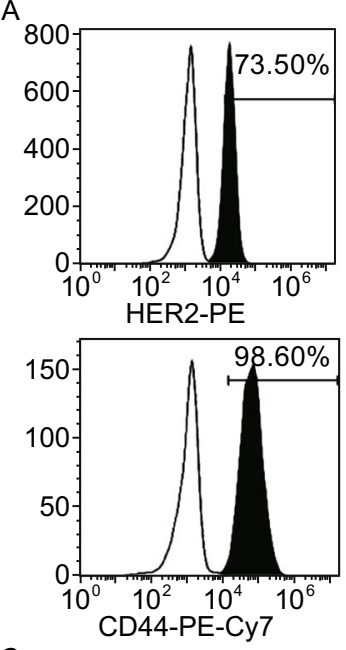

C

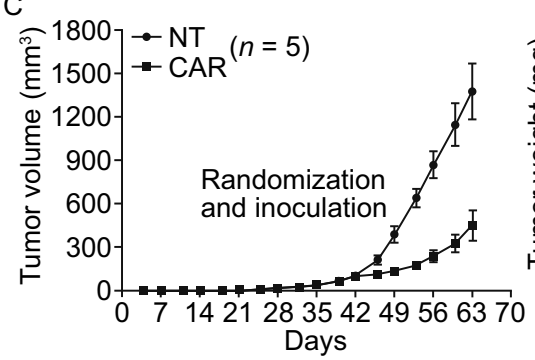

B
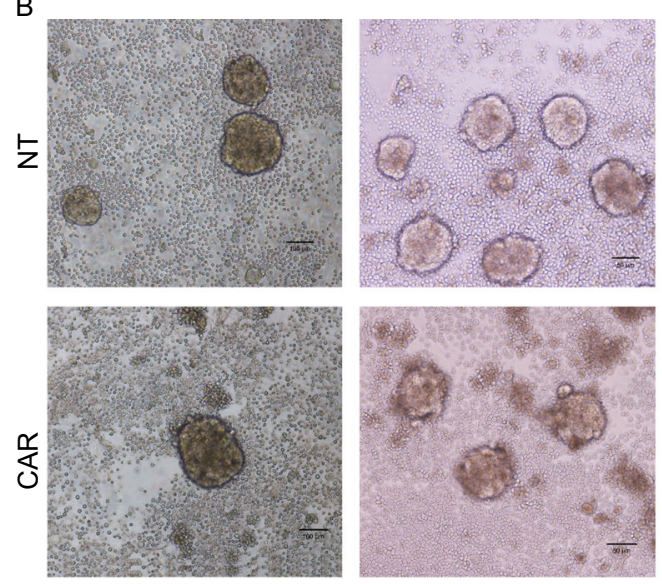

$6 \mathrm{~h}$

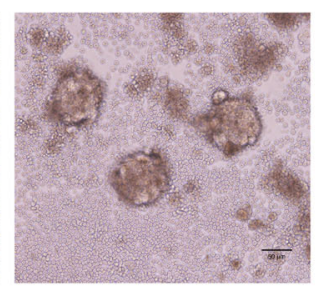

$24 \mathrm{~h}$

$(n=5)$
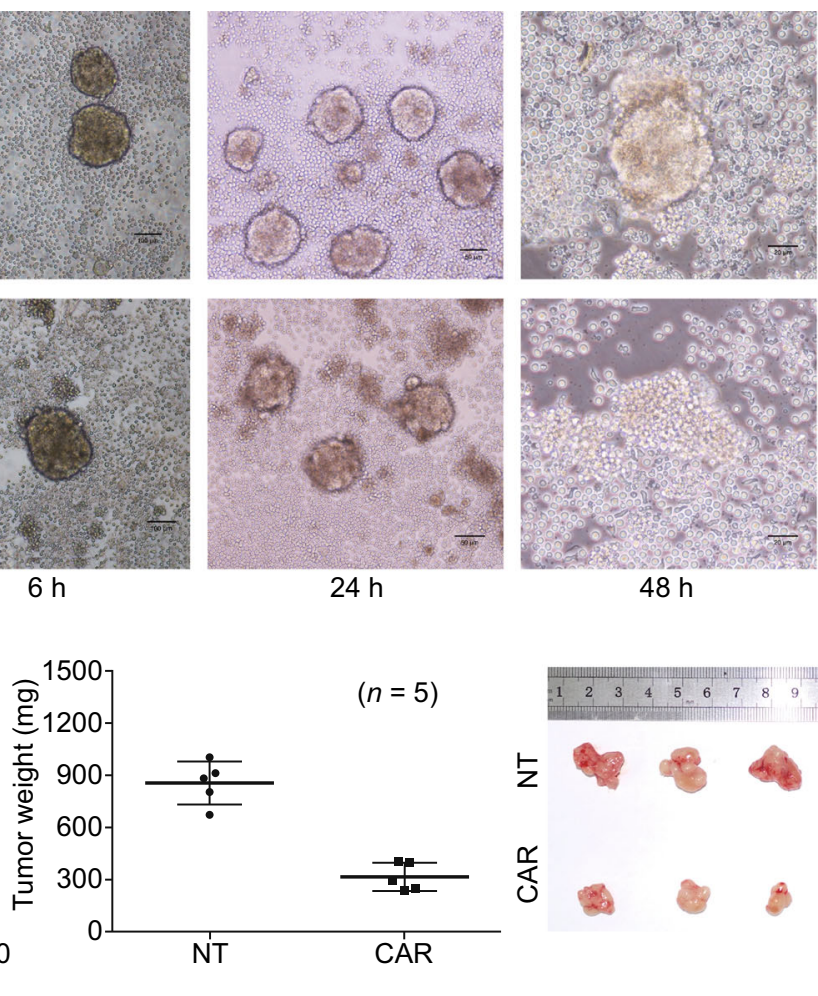

$48 \mathrm{~h}$

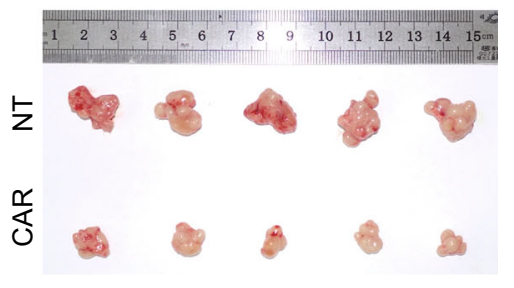

Figure 5. Effective response by CART-HER2 cells to GCSCs. (A) FACS analysis was used to determine the surface expression of HER2 and CD44 proteins in gastric cancer stem cells from suspended tumor spheres. (B) Co-culture of tumor spheres with $2 \times 10^{5}$ CART-HER2 cells, or NT T cells, per well was performed in an ultra-low adherent 24-well plate using serum-free media. Images of the killing activity, exerted by CART-HER2 cells against tumor spheres at serial time points, are shown. (C) Ten thousand gastric cancer stem cells were injected subcutaneously into the unilateral axillary region of BALB/c nude mice on day 0 . Five mice per group were randomized to the CART-HER2 and NT groups. When the mean tumor volume reached approximately $100 \mathrm{~mm}^{3} \mathrm{on}^{\mathrm{day}} 42$, intravenous treatment with T cells was performed. Mice with maximum tumor diameters over $2 \mathrm{~cm}$ were sacrificed on day 63 . The tumor volume data are represented as mean $\pm \mathrm{SD}\left(\mathrm{mm}^{3}\right)$. The tumor weight data are represented as mean $\pm \mathrm{SD}(\mathrm{mg})$. Images of tumor samples from sacrificed mice are shown.

TDM-1, have been tested in clinical GC trials, only trastuzumab is currently approved for the first-line treatment of AGC (Fanotto et al., 2016). Hence, to enhance treatment efficacy and minimize drug resistance, the HER2-directed CAR approach has been developed and validated in diverse tumor-bearing mouse models, including those of osteosarcoma (Rainusso et al., 2012), breast cancer (Sun et al., 2014), renal cancer (Schonfeld et al., 2015), and glioblastoma (Zhang et al., 2016). Recently, the first clinical study to use HER2-specific CAR-T cells in patients with HER2-positive sarcoma showed that the HER2-directed CAR approach performed well with respect to safety and efficacy (ORR, 7/17) (Ahmed et al., 2015). These data suggest that HER2 may be a suitable target for our CAR-modified T-cell immunotherapy. In this study, we demonstrated that CARTHER2 cells have high affinity for all types of GC cells, even those expressing low levels of HER2, and display an effective and persistent killing activity in HER2-positive tumor-bearing mice.

Additional costimulatory domains, incorporated into CARs, are indispensable for engineering $T$ cells that show a potent and sustained antitumor activity (Dai et al., 2016). Despite the existence of multiple alternative costimulatory molecules, the majority of modular designs, used in clinical trials, are based on the CD137 and CD28 signaling domains. Tcells expressing CD137-containing CARs are likely to show higher proliferation and persistence than those expressing CD28-containing CARs (Zhang et al., 2015). The mechanism underlying these functional differences remains unclear. One study showed that the early exhaustion of Tcells, induced at varying levels by an antigen-independent tonic signaling caused by CAR clustering, was ameliorated by CD137 domains. This may have occurred via inhibition of the expression of exhaustion-related genes and overexpression of the genes regulating 
metabolism, apoptosis, and responsiveness to hypoxia (Long et al., 2015). Similarly, another study showed the pivotal role of CD137 signaling-induced metabolic reprogramming in the enhanced persistence of CAR-T cells. These metabolic changes, including the considerable enhancements in respiratory capacity, fatty acid oxidation, and mitochondrial biogenesis, result in the differentiation of central memory and prolonged survival of CAR-T cells (Kawalekar et al., 2016). The results of these studies agree with our findings of increased proportions of $\mathrm{CD} 45 \mathrm{RO}^{+} \mathrm{CD} 62 \mathrm{~L}^{+} \mathrm{CCR} 7^{+}$cells, and long-term survival of CAR-T cells, in vivo. However, CD28 CARs exert greater cytotoxic effects on T cells than do CD137 CARs. Combining these two domains, or their ligands, results in higher antitumor efficacy in some mouse models (Zhao et al., 2015); however, this structural design warrants further clinical study.

Transduction of $C A R$ into primary T cells is accomplished using a series of gene transfer platforms, ranging from a plasmid vector- to virus-mediated gene delivery; each approach offers its pros and cons with respective efficiency, toxicity, practicability, and cost. The recombinant plasmid was initially used for the genetic modification of T cells because of safety concerns; although this method is associated with a low risk of transgene-specific immune responses and insertional mutagenesis, it delivers low transfection efficiency and limited cell survival (Jensen et al., 2000). Another approach, not involving transgene integration, is the mRNA electroporation system, which exhibits very high transfection efficiency and facilitates rapid, but transient, CAR expression in Tcells (Zhao et al., 2006). Recently, the Sleeping Beauty (SB) and PiggyBac (PB) transposon/transposase systems, which can integrate genes into target cellular genomes, were evaluated in CAR-related therapies. The low efficiency of genetic transposition is a major obstacle preventing the broad clinical application of this method; however, the low cost, convenient manufacturing, and mild toxicity of this system render it attractive as an alternative treatment strategy (Kebriaei et al., 2016). Surprisingly, one study demonstrated a markedly enhanced CAR transduction using a minicircle vector carrying an SB system (Monjezi et al., 2017). Because they are currently the most widely used vectors, retroviral and lentiviral vectors allow for the stable and efficient transfection of CAR into T cells. The ability of these modified cells to elicit a welltolerated, effective, and persistent therapeutic response has been proven in patients with different types of cancer (Jackson et al., 2016).

Tumors possess a hierarchical structure, in which CSCs serve as the tumor-initiating cells and are responsible for drug resistance, tumor progression, recurrence, and metastasis; thus eradication of CSCs is a promising method for treating cancer (Pan et al., 2015). GCSCs have also been identified using specific markers, such as CD44 (Takaishi et al., 2009); the stemness properties of GCSCs are studied using two standard approaches: the in vitro sphere-forming assay and in vivo serial tumor passaging (Brungs et al., 2016). Some studies have found that HER2 plays a critical role in maintaining the CSC subpopulation of GC cells (Jiang et al., 2012), as well as that of other tumor cells, such as breast tumor cells (Korkaya and Wicha, 2013). Therefore, because of the eradication of CSCs, HER2-targeted therapy may result in improved treatment outcomes in patients with HER2-positive GC. In this study, we used sphere cultures in vitro, and tumor formation in mice, to successfully validate the self-renewal and tumor-initiating capacity of $\mathrm{CD}_{4} 4^{+} \mathrm{HER} 2^{+}$GCSCs from primary gastric tumors. We found that CART-HER2 cells efficiently phagocytized and degraded the tumor spheres and suppressed the growth of xenograft tumors in nude mice inoculated with GCSCs. These results show that CART-HER2 cells exerted potent cytotoxic effects on GCSCs and suggest that CAR-T cells are potentially effective in preventing the recurrence and metastasis of GC. However, the practical impact of this treatment needs to be verified further in future clinical trials, given the genetic and functional heterogeneity of CSCs (Kreso and Dick, 2014) and the complexity of the tumor microenvironment (Plaks et al., 2015).

Although the therapeutic effects of CAR-T cells on hematological malignancies, especially CAR-T cells targeting CD-19, have been elucidated, the development of CARs for the treatment of solid tumors has been hindered by the paucity of optimal antigens, the inability of T cells to migrate to and infiltrate primary lesions, transgene immunogenicity and toxicity, ongoing evolution of cancer cells, possible existence of CSC populations, and the immunosuppressive tumor microenvironment. Therefore, additional methods for implementing CAR-T cell therapy against solid tumors have been explored. Modifying T cells with inducible cytokine or chemokine receptor transgenes has been shown to enhance their antitumor activity and ability to localize. For example, supplementary secretion of IL-12 by T cells, engineered with CAR and CAR-inducible $\|-12$ genes in targeted sites, improved the intrinsic functions of these $T$ cells and boosted the local immune response by facilitating the recruitment and activation of other killing cells, the initiation of innate immunity, and modulation of suppressor cells (Chmielewski et al., 2014). Similarly, synchronous blockade of PD-1 in CAR-T cells, via genetic modification, also prolonged cell survival (Cherkassky et al., 2016) and augmented tumor-killing efficacy in mouse tumor models (Liu et al., 2016). Another approach involves designing more intelligent CAR constructions to minimize the off-target effects. These constructions include bispecific CAR-T cells, which can be activated only by the identification of both CAR molecules (Wilkie et al., 2012), and the synNotch circuit, in which T cells are armed only by a synthetic Notch receptor activated by one target to induce the expression of a CAR against another target (Roybal et al., 2016). Additionally, combining CAR-T cells with ibrutinib in patients with leukemia (Fraietta et al., 2016) may represent a potential strategy for enhancing therapeutic efficacy against solid tumors.

In conclusion, we showed that HER2-directed CAR-T cells exhibit effective and persistent antitumor activity 
against patient-derived GC cells, and GCSCs, in vitro and in vivo. Our results suggest that these modified T cells may be applicable in patients with HER2-positive GC; however, the toxicity and immunogenicity of CAR need to be addressed in future clinical trials.

\section{MATERIALS AND METHODS}

Cells

The human GCCLs, N87, 7901, AGS, HGC27, MGC803, BGC823, and MKN45 were obtained from the Institute of Basic Medicine of Chinese PLA General Hospital (PLAGH). PGCCs were obtained from the Department of General Surgery of PLAGH. All GC cells were cultured in RPMI-1640 medium supplemented with $10 \%$ fetal bovine serum (FBS), except the MGC803 cells, which were cultured in DMEM (high glucose) containing 10\% FBS.

\section{Isolation of primary gastric tumor cells}

Fresh human GC tissues were obtained from patients immediately after resection. All samples were transported on ice to the laboratory within $30 \mathrm{~min}$, and were immediately mechanically disaggregated into 1-mm pieces using scissors. These tumor pieces were then digested with $1 \mathrm{mg} / \mathrm{mL}$ collagenase I and $1 \mathrm{mg} / \mathrm{mL}$ collagenase IV (Life Technologies, Waltham, MA, USA), and diluted in phosphate buffered saline (PBS) at $37^{\circ} \mathrm{C}$ for at least $1 \mathrm{~h}$. Digestion was terminated with DMEM/F12 containing 20\% FBS (Life Technologies).

\section{Tumorsphere culture and formation}

To form spheres, digested cells from primary gastric tumors were cultured at a density of $10^{4}$ cells per well in ultra-low attachment 6-well plates (Corning, NY, USA) in DMEM/F12 supplemented with 2\% B27 (Invitrogen, CA, USA), EGF (20 ng/mL, Invitrogen), bFGF (10 ng/mL, Invitrogen), LIF (10 ng/mL, Peprotech, Hartford, CT, USA), and HEPES (Invitrogen). The culture medium was replenished twice per week. Spheres with a diameter $>100 \mu \mathrm{m}$ were counted 14 days after being seeded.

\section{Constructs and lentiviral packaging}

The scFv nucleotide sequence, targeting HER2 antigens, was derived from our proprietary product (patent CN105384824A, China). The entire DNA sequence of CAR.HER2-CD137ל, which

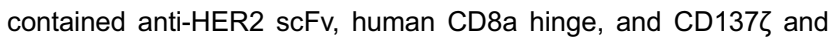
$\mathrm{CD} 3 \zeta$ signaling domains, was constructed based on our previously published CAR sequence (Dai et al., 2015). Complete clinical-grade viral particles were produced using transfection package cells with a four-plasmid system. The GFP-harboring vector CAR.HER2CD137ろ-GFP was also constructed to verify transduction efficiency.

\section{Generation and expansion of CART-HER2 cells}

Anticoagulated fresh blood was obtained from three healthy donors. PBMCs in buffy coats were purified using lymphocyte separation medium (Cedarlane), via density gradient centrifugation, after the serum was collected from the blood samples. The washed PBMCs were directly added to the lymphocyte culture medium (Takara) supplemented with the anti-CD3 mAb OKT3 (500 ng/ $/ \mathrm{L})$ and IL-2 $(300 \mathrm{U} / \mathrm{mL})$. The generation and expansion of CAR-T cells was performed in accordance with our published protocol pertaining to cytokine-induced killer (CIK) cells (Wang et al., 2013). Stable transfection of T cells with lentivirus-mediated CAR was conducted on day 2 of cell culturing. Transfection efficiency and phenotypic properties of the CAR-T cells were evaluated by FACS analysis on day 12 .

\section{HER2 knockdown by lentivirus-mediated shRNA}

Knockdown of HER2 expression in N87 and 7901 cells was performed by transfection with a lentivirus harboring human HER2specific shRNA and the GFP gene. The lentivirus was purchased from GenePharma. The target sequence for HER2 was 5'GGAAGGACATCTTCCACAAGA-3'. HER2 knockdown was confirmed by $q P C R$ and FACS analysis.

\section{Flow cytometry analysis and cell sorting}

All of the anti-human antibodies used in this study, including HER2 (phycoerythrin, PE), CD3 (chlorophyll protein complex PerCP), CD4 (fluorescein isothiocyanate, FITC), CD8-PE, CD45RO (allophycocyanin, APC), CD56-APC, CD62L-PE, CCR7-PE-Cy7, and CD44$\mathrm{PE}-\mathrm{Cy} 7$, were purchased from BD Biosciences. Isotype-matched control mAbs were applied in all the procedures. Additionally, GFPpositive HER2 ${ }^{\mathrm{KD}} \mathrm{N} 87$ and 7901 cells were separated from untransfected cells, based on the degree of fluorescence, by a BD Influx cell sorter (BD Biosciences). FACS data were analyzed by a FACSCalibur flow cytometer (BD Biosciences) and FlowJo software (Version 10.0.7, FlowJo, Ashland, OR).

\section{Cytotoxicity assays}

The specific in vitro antitumor activity of CART-HER2 cells was evaluated using a standard LDH release assay (Promega). Briefly, the different types of target cells were seeded in triplicate 96-well plates, at a density of $10^{4}$ cells per well, with $50 \mu \mathrm{L}$ of medium; then, an equal volume of effector cells, or control medium, was added to each well to ensure an E/T ratio of 20:1, 10:1, 5:1, or 1:1. After 4-h and 8-h coincubation, cell supernatants were obtained by centrifugation. Fifty microliters of sample from each well was transferred to new plates to measure the absorbance signals using a plate reader (Multiskan MK3, Thermo Scientific); the remaining samples were collected for cytokine measurements. The average cytotoxicity percentage was calculated according to the formula provided by Promega.

\section{Cytokine secretion assays}

The harvested samples of supernatant, obtained from the 4-h coincubation of effector cells with target cells at an E/T ratio of 20:1, were assessed for the levels of cytokine secretion. The concentrations of IL-2, IL-4, TNF- $\alpha$, IFN- $\gamma$, GM-CSF, and GZMB were batchmeasured using an enzyme-linked immunosorbent assay kit (BD Biosciences) in accordance with the operating manual.

\section{Quantitative real-time PCR}

To determine the levels of CAR.HER2-CD137ろ gene expression, real-time $\mathrm{PCR}$ was performed using an $\mathrm{ABI}$ PRISM 7900HT 
Sequence Detection System (Applied Biosystems) according to a procedure published previously (Till et al., 2012). A 153-base pair (bp) DNA sequence, harboring fragments of the CD8a and CD137ל moieties, was amplified using the following primers: $5^{\prime}$-GGTCCT TCTCCTGTCACTGGTT-3' and 5'-TCTTCTTCTTCTGGAAATCGG CAG-3'. The transcription level of HER2 in HER2 ${ }^{\mathrm{KD}} \mathrm{N} 87$ and 7901 cells was quantified via amplification of an 86-bp fragment using the following primers: 5'- ATATATCGAGGCGATAGGGTTAAGG-3' and 5'-CCGGGGCATATCTTCTGGAAT-3'. $\beta$-Actin was used as a control for normalization.

\section{Xenograft mouse model of GC}

The animal experiments were approved by the Animal Ethics Committee of PLAGH. BALB/c nude mice were purchased from the Beijing Vital River Laboratory Animal Technology Co. Four- to 5-week-old female BALB/c nude mice were bred under specific pathogen-free conditions in the Laboratory Animal Center of PLAGH. One hundred microliters of $2 \times 10^{6}$ HER2 $^{\text {hight }} 7901$ cells was injected subcutaneously into the unilateral axillary region of BALB/c nude mice on day 0 . The rate of xenotransplanted tumor formation was $100 \%$. At least five HER2 $2^{\text {hight }}$ tumor-bearing mice were randomly assigned to the CART-HER2 and NT groups before treatment. The TV of each mouse was measured twice weekly using a vernier caliper, and was calculated according to the following formula: $T V=1 / 2 \times$ length $\times$ width $^{2}$. On day 12 , when the mean TV reached approximately $100 \mathrm{~mm}^{3}, 200 \mu \mathrm{L}$ of $2 \times 10^{7}$ CART-HER2 cells, or NT T cells, were infused into the caudal veins of the mice. For the detection of CAR copy number, peripheral blood samples were collected from the caudal veins, or eyeballs, of HER2 $2^{\text {hight }}$ mice, treated with CARTHER2 cells, every 15 days. Inoculation with GCSCs and treatment in $\mathrm{BALB} / \mathrm{C}$ nude mice were performed as described above. The total number of injected CSCs was $1 \times 10^{4}$, and the tumor formation rate was $100 \%$. Five mice per group were randomized to the CART-HER2 and NT groups. Mice with maximum tumor diameters over $2 \mathrm{~cm}$, or $20 \%$ loss of body weight, were euthanized.

\section{Histological and immunohistochemical examinations}

Tumor tissue samples, which had been resected from sacrificed mice, were fixed in $4 \%$ paraformaldehyde for 4-6 $\mathrm{h}$, dehydrated in ethyl alcohol, and embedded in paraffin, before being cut into multiple $6-\mu \mathrm{m}$ thin sections using a microtome. HE and IHC staining were performed using standard procedures. Primary rabbit anti-human CD3 antibody (ab5690, Abcam) and a secondary biotinylated goat anti-rabbit antibody were used for IHC staining.

\section{Statistical analysis}

All results are reported as the mean \pm standard deviation (SD), and were analyzed by the unpaired $t$-test. Data were plotted using GraphPad Prism software version 6.0c for Mac OS. $P$-values $<0.05$ were considered statistically significant.

\section{ACKNOWLEDGMENTS}

The authors thank all patients who participated and their families, as well as the investigators and staff at this study for their valuable contribution to this study. We'd like to give a special thanks to
Zhiqiang Wu for the construction of plasmids. This study was supported by grants from the National Natural Science Foundation of China (Grant Nos. 81230061, 81402566, 81672319, 81602507, and 81602711), the Science and Technology Planning Project of Beijing City (No. Z151100003915076) and the key Nursery Project of Chinese PLA General Hospital (16KMZ05) and was partially supported by a grant from the National Basic Science and Development Programme of China (No. 2013BAI01B04).

\section{ABBREVIATIONS}

$\mathrm{ACl}$, adoptive cellular immunotherapy; CAR, chimeric antigen receptor; CRS, severe cytokine release syndrome; CSC, cancer stem cell; FACS, fluorescent activated cell sorting; GC, gastric cancer; GFP, green fluorescence protein; HE, hematoxylin-eosin staining; HER2, epidermal growth factor receptor 2; IHC, immunohistochemical staining; $\mathrm{LDH}$, lactate dehydrogenase; ORR, overall response rate; PBMC, peripheral blood mononuclear cell; scFv, single chain variable fragment.

\section{COMPLIANCE WITH ETHICS GUIDELINES}

Yanjing Song, Chuan Tong, Yao Wang, Yunhe Gao, Hanren Dai, Yelei Guo, Xudong Zhao, Yi Wang, Zizheng Wang, Weidong Han, and Lin Chen declare that they have no conflict of interest.

All procedures followed were in accordance with the ethical standards of the responsible committee on human experimentation (institutional and national) and with the Helsinki Declaration of 1975 , as revised in 2000. Informed consent was obtained from all patients for being included in the study.

All institutional and national guidelines for the care and use of laboratory animals were followed.

\section{OPEN ACCESS}

This article is distributed under the terms of the Creative Commons Attribution 4.0 International License (http://creativecommons.org/ licenses/by/4.0/), which permits unrestricted use, distribution, and reproduction in any medium, provided you give appropriate credit to the original author(s) and the source, provide a link to the Creative Commons license, and indicate if changes were made.

\section{REFERENCES}

Ahmed N, Brawley VS, Hegde M, Robertson C, Ghazi A, Gerken C, Liu E, Dakhova O, Ashoori A, Corder A et al (2015) Human Epidermal Growth Factor Receptor 2 (HER2)-specific chimeric antigen receptor-modified $T$ cells for the immunotherapy of HER2-positive sarcoma. J Clin Oncol 33:1688-1696

Brungs D, Aghmesheh M, Vine KL, Becker TM, Carolan MG, Ranson M (2016) Gastric cancer stem cells: evidence, potential markers, and clinical implications. J Gastroenterol 51:313-326

Chen W, Zheng R, Baade PD, Zhang S, Zeng H, Bray F, Jemal A, Yu $X Q$, He J (2016) Cancer statistics in China, 2015. CA Cancer J Clin 66:115-132 
Cherkassky L, Morello A, Villena-Vargas J, Feng Y, Dimitrov DS, Jones DR, Sadelain M, Adusumilli PS (2016) Human CAR T cells with cell-intrinsic PD-1 checkpoint blockade resist tumor-mediated inhibition. J Clin Invest 126:3130-3144

Chmielewski M, Hombach AA, Abken H (2014) Of CARs and TRUCKs: chimeric antigen receptor (CAR) T cells engineered with an inducible cytokine to modulate the tumor stroma. Immunol Rev 257:83-90

Dai H, Zhang W, Li X, Han Q, Guo Y, Zhang Y, Wang Y, Wang C, Shi F, Zhang $Y$ et al (2015) Tolerance and efficacy of autologous or donor-derived T cells expressing CD19 chimeric antigen receptors in adult B-ALL with extramedullary leukemia. Oncoimmunology 4:e1027469

Dai H, Wang Y, Lu X, Han W (2016) Chimeric antigen receptors modified T-cells for cancer therapy. J Natl Cancer Inst 108:djv439

Eshhar Z, Waks T, Gross G, Schindler DG (1993) Specific activation and targeting of cytotoxic lymphocytes through chimeric single chains consisting of antibody-binding domains and the gamma or zeta subunits of the immunoglobulin and T-cell receptors. Proc Natl Acad Sci USA 90:720-724

Fanotto V, Ongaro E, Rihawi K, Avallone A, Silvestris N, Fornaro L, Vasile E, Antonuzzo L, Leone F, Rosati G et al (2016) HER-2 inhibition in gastric and colorectal cancers: tangible achievements, novel acquisitions and future perspectives. Oncotarget 7:69060-69074

Fraietta JA, Beckwith KA, Patel PR, Ruella M, Zheng Z, Barrett DM, Lacey SF, Melenhorst JJ, McGettigan SE, Cook DR et al (2016) Ibrutinib enhances chimeric antigen receptor T-cell engraftment and efficacy in leukemia. Blood 127:1117-1127

Jackson HJ, Rafiq S, Brentjens RJ (2016) Driving CAR T-cells forward. Nat Rev Clin Oncol 13:370-383

Jensen MC, Clarke P, Tan G, Wright C, Chung-Chang W, Clark TN, Zhang F, Slovak ML, Wu AM, Forman SJ et al (2000) Human T lymphocyte genetic modification with naked DNA. Mol Ther 1:49-55

Jiang J, Zhang Y, Chuai S, Wang Z, Zheng D, Xu F, Zhang Y, Li C, Liang Y, Chen $Z$ (2012) Trastuzumab (herceptin) targets gastric cancer stem cells characterized by CD90 phenotype. Oncogene 31:671-682

Kawalekar OU, O'Connor RS, Fraietta JA, Guo L, McGettigan SE, Posey AD Jr, Patel PR, Guedan S, Scholler J, Keith B et al (2016) Distinct signaling of coreceptors regulates specific metabolism pathways and impacts memory development in CAR T cells. Immunity 44:380-390

Kebriaei P, Singh H, Huls MH, Figliola MJ, Bassett R, Olivares S, Jena B, Dawson MJ, Kumaresan PR, Su S et al (2016) Phase I trials using sleeping beauty to generate CD19-specific CAR T cells. J Clin Invest 126:3363-3376

Korkaya H, Wicha MS (2013) HER2 and breast cancer stem cells: more than meets the eye. Cancer Res 73:3489-3493

Kreso A, Dick JE (2014) Evolution of the cancer stem cell model. Cell Stem Cell 14:275-291

Liu X, Ranganathan R, Jiang S, Fang C, Sun J, Kim S, Newick K, Lo A, June $\mathrm{CH}$, Zhao $\mathrm{Y}$ et al (2016) A chimeric switch-receptor targeting PD1 augments the efficacy of second-generation CAR T cells in advanced solid tumors. Cancer Res 76:1578-1590

Long AH, Haso WM, Shern JF, Wanhainen KM, Murgai M, Ingaramo M, Smith JP, Walker AJ, Kohler ME, Venkateshwara VR et al
(2015) 4-1BB costimulation ameliorates T cell exhaustion induced by tonic signaling of chimeric antigen receptors. Nat Med 21:581590

Monjezi R, Miskey C, Gogishvili T, Schleef M, Schmeer M, Einsele H, Ivics Z, Hudecek M (2017) Enhanced CAR T-cell engineering using non-viral Sleeping Beauty transposition from minicircle vectors. Leukemia 31:186-194

Pan Q, Li Q, Liu S, Ning N, Zhang X, Xu Y, Chang AE, Wicha MS (2015) Concise review: targeting cancer stem cells using immunologic approaches. Stem Cells 33:2085-2092

Park JH, Geyer MB, Brentjens RJ (2016) CD19-targeted CAR T-cell therapeutics for hematologic malignancies: interpreting clinical outcomes to date. Blood 127:3312-3320

Plaks V, Kong N, Werb Z (2015) The cancer stem cell niche: how essential is the niche in regulating stemness of tumor cells? Cell Stem Cell 16:225-238

Porter DL, Levine BL, Kalos M, Bagg A, June CH (2011) Chimeric antigen receptor-modified $T$ cells in chronic lymphoid leukemia. N Engl J Med 365:725-733

Rainusso N, Brawley VS, Ghazi A, Hicks MJ, Gottschalk S, Rosen JM, Ahmed N (2012) Immunotherapy targeting HER2 with genetically modified $\mathrm{T}$ cells eliminates tumor-initiating cells in osteosarcoma. Cancer Gene Ther 19:212-217

Roybal KT, Rupp LJ, Morsut L, Walker WJ, McNally KA, Park JS, Lim WA (2016) Precision tumor recognition by $T$ cells with combinatorial antigen-sensing circuits. Cell 164:770-779

Schonfeld K, Sahm C, Zhang C, Naundorf S, Brendel C, Odendahl M, Nowakowska P, Bonig H, Kohl U, Kloess S et al (2015) Selective inhibition of tumor growth by clonal NK cells expressing an ErbB2/HER2-specific chimeric antigen receptor. Mol Ther 23:330-338

Sun M, Shi H, Liu C, Liu J, Liu X, Sun Y (2014) Construction and evaluation of a novel humanized HER2-specific chimeric receptor. Breast Cancer Res 16:R61

Takaishi S, Okumura T, Tu S, Wang SS, Shibata W, Vigneshwaran R, Gordon SA, Shimada Y, Wang TC (2009) Identification of gastric cancer stem cells using the cell surface marker CD44. Stem Cells 27:1006-1020

Teachey DT, Lacey SF, Shaw PA, Melenhorst JJ, Maude SL, Frey N, Pequignot E, Gonzalez VE, Chen F, Finklestein J et al (2016) Identification of predictive biomarkers for cytokine release syndrome after chimeric antigen receptor T-cell therapy for acute lymphoblastic leukemia. Cancer Discov 6:664-679

Ter Veer E, Mohammad NH, Lodder P, Ngai LL, Samaan M, van Oijen MG, van Laarhoven HW (2016) The efficacy and safety of S-1-based regimens in the first-line treatment of advanced gastric cancer: a systematic review and meta-analysis. Gastric Cancer 19:696-712

Thibault C, Khodari W, Lequoy M, Gligorov J, Belkacemi Y (2013) HER2 status for prognosis and prediction of treatment efficacy in adenocarcinomas: a review. Crit Rev Oncol Hematol 88:123133

Till BG, Jensen MC, Wang J, Qian X, Gopal AK, Maloney DG, Lindgren CG, Lin Y, Pagel JM, Budde LE et al (2012) CD20specific adoptive immunotherapy for lymphoma using a chimeric antigen receptor with both CD28 and 4-1BB domains: pilot clinical trial results. Blood 119:3940-3950 
Vakiani E (2015) HER2 testing in gastric and gastroesophageal adenocarcinomas. Adv Anat Pathol 22:194-201

Van Cutsem E, Sagaert X, Topal B, Haustermans K, Prenen H (2016) Gastric cancer. Lancet 388:2654-2664

Wang Y, Bo J, Dai HR, Lu XC, Lv HY, Yang B, Wang T, Han WD (2013) ClK cells from recurrent or refractory AML patients can be efficiently expanded in vitro and used for reduction of leukemic blasts in vivo. Exp Hematol 41(241-252):e243

Wilkie S, van Schalkwyk MC, Hobbs S, Davies DM, van der Stegen SJ, Pereira AC, Burbridge SE, Box C, Eccles SA, Maher J (2012) Dual targeting of ErbB2 and MUC1 in breast cancer using chimeric antigen receptors engineered to provide complementary signaling. J Clin Immunol 32:1059-1070

Zhang T, Cao L, Xie J, Shi N, Zhang Z, Luo Z, Yue D, Zhang Z, Wang L, Han W et al (2015) Efficiency of CD19 chimeric antigen receptor- modified T cells for treatment of B cell malignancies in phase I clinical trials: a meta-analysis. Oncotarget 6:33961-33971

Zhang C, Burger MC, Jennewein L, Genssler S, Schonfeld K, Zeiner P, Hattingen E, Harter PN, Mittelbronn M, Tonn T et al (2016) ErbB2/HER2-Specific NK Cells for Targeted Therapy of Glioblastoma. J Natl Cancer Inst 108:djv375

Zhao Y, Zheng Z, Cohen CJ, Gattinoni L, Palmer DC, Restifo NP, Rosenberg SA, Morgan RA (2006) High-efficiency transfection of primary human and mouse T lymphocytes using RNA electroporation. Mol Ther 13:151-159

Zhao Z, Condomines M, van der Stegen SJ, Perna F, Kloss CC, Gunset G, Plotkin J, Sadelain M (2015) Structural design of engineered costimulation determines tumor rejection kinetics and persistence of CAR T cells. Cancer Cell 28:415-428 